\title{
AN INTEGRAL OVER THE INTERIOR OF A SIMPLEX
}

\author{
by HENRY JACK
}

(Received 3rd June, 1962)

\section{Introduction}

If $f(z)$ is analytic in a suitable domain, it is shown how the integral of $f\left(\alpha_{1} x_{1}+\ldots+\alpha_{n} x_{n}\right)$ over the interior of a simplex may be reduced to the evaluation of a contour integral, in fact to an exercise in partial fractions.

The contour integral is expressed in two ways, according as the simplex is given in terms of its vertices or faces.

\section{Notation}

It will be convenient to use a matrix notation. Let $x=\left\{x_{1}, \ldots, x_{n}\right\}$ be a column vector representing a point in real $n$-dimensional Euclidean space, where the volume element is $d x=\prod_{r=1}^{n} d x_{r}$. Using a dash for the transpose let $\alpha^{\prime}=\left(\alpha_{1}, \ldots, \alpha_{n}\right)$ be a row vector of real or complex constants.

Suppose that the co-ordinate vectors of the vertices of a simplex are

$$
v_{r}=\left\{x_{r 1}, \ldots, x_{r s}, \ldots, x_{r n}\right\}(1 \leqq r \leqq n+1),
$$

and define $n+1$ numbers by

$$
z_{r}=\alpha^{\prime} v_{r}=\sum_{s=1}^{n} \alpha_{s} x_{r s}(1 \leqq r \leqq n+1)
$$

\section{Two Lemmas}

- Lemma 1. Let $S^{*}$ be the simplex $x_{1} \geqq 0, x_{2} \geqq 0, \ldots, x_{n} \geqq 0, x_{1}+x_{2}+\ldots+x_{n} \leqq k$; then

$$
\int_{S^{*}} \exp \left(\alpha^{\prime} x\right) d x=\frac{1}{2 \pi i} \int_{\Gamma} \frac{e^{k z} d z}{z \prod_{r=1}^{n}\left(z-\alpha_{r}\right)},
$$

where $\Gamma$ is a contour enclosing $z=0, \alpha_{1}, \ldots, \alpha_{n}$.

Proof. See Lemma 1 of (1).

Lemma 2. If $V_{n}$ is the volume of $S$, the simplex with vertices (1), and $C_{1}$ is a contour enclosing all the $z_{r}$ of $(2)$, then

$$
\int_{S} \exp \left(\alpha^{\prime} x\right) d x=\frac{n ! V_{n}}{2 \pi i} \int_{C_{1}} \frac{e^{z} d z}{\prod_{r=1}^{n+1}\left(z-z_{r}\right)}
$$


Proof. Make the change of variables $y=x-v_{n+1}$; then $S$ becomes a simplex $S_{0}$ with one vertex at the origin and the remaining vertices at

$$
w_{r}=v_{r}-v_{n+1}(1 \leqq r \leqq n)
$$

and the left-hand side of (3) becomes

$$
e^{z_{n+1}} \int_{S_{0}} \exp \left(\alpha^{\prime} y\right) d y
$$

If now $W$ is the $n \times n$ matrix whose $r$ th column is $w_{r}$, then the determinant $|W|$ of $W$ is just $\pm n ! V_{n}$ (see (2), page 124). Apply the transformation $y=W x$ to (5); then $S_{0}$ becomes the simplex $S^{*}$ of Lemma 1 , with $k=1$, and (5) becomes

$$
n ! V_{n} e^{z_{n+1}} \int_{s^{*}} \exp \left(\alpha^{\prime} W x\right) d x
$$

Let $\beta_{r}$ be the $r$ th component of the row vector $\alpha^{\prime} W$. Then, by Lemma 1, with $k=1$, (6) becomes, for a contour $\Gamma$ enclosing $\zeta=0, \beta_{1}, \ldots, \beta_{n}$,

$$
\frac{n ! V_{n}}{2 \pi i} \int_{\Gamma} \frac{\exp \left(\zeta+z_{n+1}\right) d \zeta}{\zeta \prod_{r=1}^{n}\left(\zeta-\beta_{r}\right)}
$$

The lemma now follows on letting $z=\zeta+z_{n+1}$, since

$$
\beta_{r}+z_{n+1}=\alpha^{\prime} w_{r}+\alpha^{\prime} v_{n+1}=\alpha^{\prime} v_{r}=z_{r} \quad(1 \leqq r \leqq n) .
$$

\section{The Main Theorem}

Theorem 1. Suppose that $f(z)=\sum_{r=0}^{\infty} c_{r} z^{r}$ for $|z|<R$, and that

$$
F_{s}(z)=\frac{1}{(s-1) !} \int_{0}^{z} f(t)(z-t)^{s-1} d t \quad(s=1,2, \ldots) .
$$

Let $S$ be the simplex with vertices (1). Suppose further that the numbers $z_{r}$ of (2) satisfy $\left|z_{r}\right|<R_{1}$ for some $R_{1}<R$, and let $C$ be the circle $|z|=\rho$, where $R_{1}<\rho<R$. Then

where

$$
\int_{S} f\left(\alpha^{\prime} x\right) d x=\int_{C} F_{n}(z) K_{n}(z) d z
$$

$$
K_{n}(z)=\frac{n ! V_{n}}{2 \pi i} \frac{1}{\prod_{r=1}^{n+1}\left(z-z_{r}\right)} .
$$

Proof. In (3) of Lemma 2, replace for $\lambda>1, \alpha_{r}$ by $\lambda \alpha_{r}, z$ by $\lambda z$, and let the contour $C_{1}$ become a contour $C_{2}$.

Then since

$$
\int_{C_{2}} z^{m} K_{n}(z) d z=0 \text { for } m \leqq n-1,
$$


on expanding both exponentials and equating corresponding powers of $\lambda$, it follows that

$$
\int_{S}\left(\alpha^{\prime} x\right)^{r} d x=\frac{r !}{(n+r) !} \int_{C_{2}} z^{n+r} K_{n}(z) d z .
$$

Now choose $C_{2}$ to be the circle $C$, multiply both sides of (9) by the $c_{r}$ and sum the resulting series. Then

$$
\int_{S} f\left(\alpha^{\prime} x\right) d x=\int_{C} F_{n}(z) K_{n}(z) d z,
$$

where

$$
\begin{aligned}
F_{n}(z) & =\sum_{r=0}^{\infty} \frac{r ! c_{r} z^{n+r}}{(n+r) !}=\sum_{r=0}^{\infty} c_{r} \int_{0}^{z} \frac{t^{r}(z-t)^{n-1}}{(n-1) !} d t \\
& =\frac{1}{(n-1) !} \int_{0}^{z} f(t)(z-t)^{n-1} d t,
\end{aligned}
$$

as in the enunciation. The circle $C$ may now be expanded into any contour in the domain of regularity of $F_{n}(z)$. Because of remark $(8) F_{n}(z)$ need only be evaluated to within a polynomial of degree $n-1$.

In the case when the row vector $\alpha^{\prime}$ is real and the $z_{r}$ are all distinct there is a geometrical interpretation of the contour integral. Project the vertices $V_{1}, \ldots$, $V_{n+1}$ of $S$ orthogonally onto a set of points $P_{1}, \ldots, P_{n+1}$ along the vector $\alpha^{\prime}$. Let $D_{r}$ be the product of the signed distances from the remaining points $P$ to $P_{r}$, then the multiple integral of (7) is

$$
n ! V_{n} \sum_{r=1}^{n+1} \frac{F_{n}\left(P_{r}\right)}{D_{r}}
$$

When the $z_{r}$ are not distinct, a result of Turnbull (3) shows how the contour integral of (7) may be expressed in terms of a determinant which involves the $F_{1}(z), \ldots, F_{n}(z)$.

\section{Alternative Form of the Main Theorem}

Suppose that the simplex $S$ of Theorem 1 is defined as the region bounded by the $n+1$ hyperplanes

$$
\begin{aligned}
a_{11} x_{1}+\ldots+a_{1 n} x_{n}+a_{1, n+1} & =0, \\
a_{n+1,1} x_{1}+\ldots+a_{n+1, n} x_{n}+a_{n+1, n+1} & =0 .
\end{aligned}
$$

We show in this paragraph how the $K_{n}(z)$ of Theorem 1 can be expressed in terms of $z, \alpha_{1}, \ldots, \alpha_{n}$ and the $a_{r s}$ of (10). To do this it will be convenient to denote by $A=\left(a_{r s}\right)$ the $(n+1) \times(n+1)$ matrix of coefficients of $(10)$, and by $A_{r s}$, the $n \times n$ determinant left when the row $r$ and column $s$ of $|A|$ are deleted. 
Lemma 3. The volume $V_{n}$ of the simplex (10) is given by

$$
n ! V_{n}= \pm \frac{|A|^{n}}{\prod_{r=1}^{n+1} A_{r, n+1}}
$$

Proof. Let the co-ordinates of the vertex $v_{r}=\left(x_{r 1}, \ldots, x_{r s}, \ldots, x_{r n}\right)$ be the solution of (10) when the $r$ th equation is omitted, then by Cramer's rule, allowing for an interchange of columns in the numerator, we have

$$
x_{r s}=(-)^{n+s+1} \frac{A_{r s}}{A_{r, n+1}} .
$$

Denote by $\Delta$ the $(n+1) \times(n+1)$ matrix whose $r$ th row is $\left(x_{r 1}, \ldots, x_{r n}, 1\right)$ and consider the product $\Delta A^{\prime}$. Since $v_{r}$ is the solution of (10) with the $r$ th equation omitted, every element in the $r$ th row of $\Delta A^{\prime}$ is zero, except that in the $r$ th place, where the entry is

but, by (12), this is

$$
\sum_{s=1}^{n} x_{r s} a_{r s}+a_{r, n+1}
$$

$$
\frac{(-)^{n+r+1}}{A_{r, n+1}}\left\{\sum_{s=1}^{n}(-)^{r+s} a_{r s} A_{r s}+(-)^{n+r+1} a_{r, n+1} A_{r, n+1}\right\}=\frac{(-)^{n+r+1}}{A_{r, n+1}}|A| .
$$

Thus

$$
|\Delta \| A|= \pm \frac{|A|^{n+1}}{\prod_{r=1}^{n+1} A_{r, n+1}} .
$$

The lemma follows, since $n ! V_{n}= \pm|\Delta|$ (see (2) p. 124).

Theorem 2. If $A_{(r)}(z)$ is the determinant of the matrix formed from $A$ by replacing the rth row by $\left(\alpha_{1}, \ldots, \alpha_{n},-z\right)$, then

$$
2 \pi i K_{n}(z)= \pm \frac{|A|^{n}}{\prod_{r=1}^{n+1} A_{(r)}(z)},
$$

the sign \pm being chosen to make $\pm(-)^{\frac{1}{(n+1)(n+2)}} \frac{|A|^{n}}{n+1}$ positive.

$$
\prod_{r=1}^{n+1} A_{r, n+1}
$$

Proof. Expanding $A_{(r)}(z)$ by its $r$ th row, we get

and so, by (12),

$$
A_{(r)}(z)=\sum_{s=1}^{n}(-)^{r+s} \alpha_{s} A_{r s}+(-)^{r+n+1}(-z) A_{r, n+1}
$$

$$
A_{(r)}(z)=(-)^{r+n} A_{r, n+1}\left(z-\sum_{s=1}^{n} \alpha_{s} x_{r s}\right)=(-)^{r+n} A_{r, n+1}\left(z-z_{r}\right)
$$

The theorem follows from this and Lemma 3 , apart from the choice of sign. This is determined by putting $\alpha_{1}=\alpha_{2}=\ldots=\alpha_{n}=0$ and $z=1$ in (13). 


\section{REFERENCES}

(1) H. JACK and A. M. MACBEATH, The volume of a certain set of matrices, Proc. Cambridge Phil. Soc. 55 (1959), 213-223.

(2) D. M. Y. SOMMERville, An introduction to the geometry of $\mathrm{N}$ dimensions (London, 1929).

(3) H. W. TuRngull, Note on partial fractions and determinants, Proc. Edinburgh Math. Soc. (2) 1 (1927-29), 49-54.

QuEEN'S COLLEGE

DUNDEE 